\title{
TAGUNG
}

\section{Die Europäischen Parteien: Zwischen Erweiterung und Selbstbehauptung}

\author{
Jane Öispü*
}

Das europäische Parteiensystem hat seit den 1970er Jahren, angestoßen durch die Gründung europäischer Parteiföderationen und die erste Direktwahl zum Europäischen Parlament, eine beachtliche Entwicklung erfahren. Dennoch: während die Fraktionen im Europäschen Parlament mittlerweile als parteipolitische Akteure auf europäischer Ebene anerkannt sind, werden die europäischen Parteiorganisationen weniger deutlich wahrgenommen und ihr Parteiencharakter als zweite Erscheinungsform europäischer Parteien gelegentlich in Frage gestellt. ${ }^{1}$ Neuere Entwicklungen haben die Rahmenbedingungen für das Wirken und die Entwicklung europäischer Parteien bemerkenswert geändert: erstens brachte die Osterweiterung der Union eine beachtliche Erweiterung der Mitgliederbasis europäischer Parteien mit sich. Zweitens wurden mit dem Erlass eines Parteienstatuts im November 2003 zum ersten Mal die Finanzierung und rechtliche Grundlagen europäischer Parteien geregelt. Die aktuellen Entwicklungen des europäischen Parteiensystems waren der Anlass für die internationale Konferenz „The Future European Party System“ vom 7. bis 9. Juli 2005 in Tübingen. Ferner bot ein im Jahr 2004 am Lehrstuhl von Professor Rudolf Hrbek (Universität Tübingen) angestoßenes Buchprojekt über die europapolitischen Aktivitäten politischer Parteien in den zehn neuen EU-Mitgliedstaaten ${ }^{2}$ einen weiteren Beweggrund für die Konferenz. Die in diesem Rahmen begonnene gute Zusammenarbeit mit Parteienforschern aus Mittel- und Osteuropa

\section{The Future European Party System}

Internationale Konferenz des Arbeitskreises Europäische Integration, Berlin, mit Unterstuitzung der Europäischen Kommission

Universität Tübingen, 7.-9. Juli 2005

\section{Wissenschaftliche Leitung}

Prof. Dr. Rudolf HRBEK, Universität Tübingen

Panel I: Party groups in the European Parliament

Cohesion of party groups (MEPs voting behaviour until 2004/from 2004)

Bernhard PATRY, Universität Tübingen, Institut für Politikwissenschaft

Party cohesion and voting behaviour in new member states and their implications for the cohesion of EP party groups

Kai-Olaf LANG, SWP, Berlin; Evald MIKKEL, University of Tartu, Estonia; Jan ROVNY, College of Europe, Bruges; Dr. Peter ZERVAKIS, Bertelsmann Stiftung

Alliances and „Coalitions“

Dr. Andreas HELLE, Secretariate General, PES, Brussels; Christian KREMER, Deputy Secretary-General, EPP, Brussels

MEPs and their national parties

Dr. Gail MCELROY, Trinity College Dublin; Dr. Janis IKSTENS, University of Latvia, Riga; Mindaugas JURKYNAS, University of Vilnius, Lithuania

* Jane Õispuu, M. A., Doktorandin am Lehrstuhl Prof. Dr. Rudolf Hrbek, Universität Tübingen.

1 So beispielsweise bei der vom Institut für soziale Bewegungen in Bochum veranstalteten Tagung „Europäische_Parteien als Integrationsmotoren?" am 7. und 8. November 2005.

2 Rudolf Hrbek (Hrsg.): European Parliament Elections 2004 in the Ten New EU Member States. Towards the Future European Party System, Baden-Baden 2005. 
sollte bei einer Tagung weitergeführt und vertieft werden. Mit Blick auf den gewünschten Praxisbezug waren neben Wissenschaftlern aus alten und neuen Mitgliedstaaten auch Vertreter europäischer Parteiorganisationen eingeladen.

Das Europäische Parlament im Jahre 2005 Parteienvielfalt und / oder Effektivität?

Der erste und umfangreichste Themenblock der Tagung konzentrierte sich auf die Fraktionen im Europäischen Parlament - auf die parteipolitischen Akteure in der Union schlechthin. Die Fraktionen als die am stärksten institutionalisierte Form europäischer Parteien sind dem Forscher auch analytisch-empirisch besser zugänglich - ersichtlich an der umfangreichen Forschung zu diesem Thema. Einem in diesem Zusammenhang vergleichsweise wenig behandelten Fragenkomplex widmete sich Bernhard Patry. Er ging der Frage nach, inwiefern die Osterweiterung einen Einfluss auf die innere Kohäsion der Fraktionen hatte. Sein Befund mag in Anbetracht der gewachsenen Heterogenität des Parlaments (161 nationale Delegationen gegenüber den früheren 120) überraschen: der Index of Agreement ${ }^{3}$ zeigt kaum eine Veränderung zu der Zeit vor Juni 2004. Diese Bilanz sei unter anderem auf den hohen Anteil polnischer und tschechischer Abgeordneten zurückzuführen (54 beziehungsweise 24 von insgesamt 106 neuen Abgeordneten). Da die dortigen Volksvertretungen hohe Kohäsionswerte aufweisen, könne man von einer Art bottom-up-Europäisierung sprechen. Bei einigen Fraktionen, wie beispielsweise den Europäischen Sozialdemokraten (SPE), hat Patry besonders hohe Kohäsionswerte festgestellt, was mit einer höheren Institutionalisierung der Fraktionsarbeit zusammenhinge. Vergleicht man das Verhalten von Fraktionen in verschiedenen Politikbereichen, so lautet der Befund: die Kohäsionswerte einzelner Fraktionen variieren bei außenpolitischen Themen

\section{Panel II: EU Party Organizations}

Impact on member parties at national level Dr. Robert LADRECH, Keele University; Prof. Dr. Luciano BARDI, Pisa University

Consequences resulting from EU-Enlargement

Prof. David HANLEY PhD, Cardiff University

The new Statute for European political parties: incentive for strengthening (the formation of) European parties?

Prof. Dr. Rudolf HRBEK, Univeristät Tübingen, Institut für Politikwissenschaft

Panel III: The Party Factor in EU-Institutions and in Interinstitutional Relations

Commission, Council, Committee of the Regions

Prof. Christopher LORD PhD, University of Reading

Parties as a framework for the preparation of joint positions and decisions

Dr. Andreas HELLE, Secretariate General, PES, Brussels

Panel IV: Future research on European Parties

Roundtable discussion

Moderator: Prof. Dr. Rudolf HRBEK

beachtlich. Zusammenfassend ließe sich somit festhalten, dass die Abgeordneten aus den neuen Mitgliedstaaten - entgegen anderslautender Befürchtungen - bisher keine destabilisierende Wirkung auf die Kohäsion der EPFraktionen gehabt haben, wenngleich dieser Befund bei außenpolitischen Themen etwas an Überzeugungskraft verliert.

$\mathrm{Zu}$ einem ähnlichen Thema, allerdings aus der Perspektive der neuen Mitgliedstaaten, sprach Kai-Olaf Lang. Lang ging auf verschiedene strukturelle und ideologische Spezifika der osteuropäischen Parteiensysteme ein, um

3 Der Index of Agreement dient zur Bemessung der Kohäsion und wird anhand der Relation der abgegebenen „Ja“- und „Nein“-Stimmen und der Enthaltungen bei roll call-votes ermittelt. Der Indexwert bewegt sich zwischen Eins (vollkommene Kohäsion) und Null (fehlende Kohäsion). 
dann auf deren Rückwirkungen auf die Fraktionen im Europäischen Parlament zu sprechen zu kommen. Seiner Einschätzung nach könnten vor allem strukturelle Mängel osteuropäischer Parteiensysteme - Instabilität und Heterogenität, verbunden mit hoher Volatilität und einer großen Zahl an Neugründungen - Inkohärenz hervorrufen. Die Vielfalt an Spaltungs- und Fusionsprozessen in osteuropäischen Parteienlandschaften sei jedoch nicht programmatisch bedingt, sondern ergebe sich aus starker Personalisierung der Politik und dem so genannten Big-Chief-Syndrom (Stichwort: Führer-Parteien). Als ideologischen Faktor hob Lang die große Zahl an Non-Standard-Parteien hervor, die europäische Parteien vor Schwierigkeiten stellten: so sind viele osteuropäische Parteien keiner traditionellen Parteifamilie zuzuordnen, wie beispielsweise die linkspopulistische Smer (Slowakei). Andererseits weisen viele Parteien sehr spezifische ideologische Profile und Präferenzen auf. Auch ideologische Disparitäten sowie die Schwäche grüner Parteien in Osteuropa hätten Konsequenzen für das europäische Parteiensystem. Fazit: es sind große Anstrengungen europäischer Parteien nötig, um die ,Neuen' zu integrieren, ohne den eigenen inneren Zusammenhalt zu gefährden. Die entscheidende Frage dabei ist: welche Seite beeinflusst die andere mehr und auf welche Weise? Bislang zeige sich jedoch, dass die Erweiterung entgegen mancher Befürchtung keine großen Probleme mit sich gebracht hat und die Europäisierung osteuropäischer Parteien insgesamt gut verläuft.

Einen unmittelbaren und spannenden Einblick in das ,Innenleben ' der europäischen Parteiorganisationen - den so genannten Europarteien - ermöglichten die Beiträge von Andreas Helle und Christian Kremer. Dabei fand ein Großteil der seitens der Wissenschaftler angesprochenen Themen auch bei den Praktikern Bestätigung. So betonte Helle, dass die Sozialdemokratische Partei Europas (SPE) nach der Osterweiterung mit innerer Heterogenität zu kämpfen habe: die Koordination politischer Positionen und die Kompromissfindung seien schwieriger geworden. Dabei seien insbesondere Politiker neuer Mitgliedstaaten nationalen Denkmustern verhaftet. Auch sei es schwer, sie in Debatten über policy-Inhalte einzubeziehen, was mit der Schwäche der Ideologien und personalisierten Politikstilen zusammenhinge. Eine Aufgabe der SPE sei darin $\mathrm{zu}$ sehen, die ,Neuen“ nicht als einen Block, sondern als unterschiedliche Akteure mit jeweils eigenen politischen Prioritäten wahrzunehmen (so beispielsweise die Russland-Politik von Polen und den baltischen Staaten). Von einem langen Lernprozess sprach auch Christian Kremer, auch wenn die Ausgangsposition der Europäischen Volkspartei (EVP) in ideologischer Hinsicht günstiger gewesen sei. So hätten die Christdemokraten in vielen Ländern, wie beispielsweise den baltischen Staaten, einfacher Verbündete finden können. Auch hätte sich die ,Flexibilitätsstrategie' gelohnt, trotz der Ausweitung der programmatischen Basis der Partei. Beide Praktiker bestätigten die Schwierigkeit der Koalitionsbildungsprozesse auf europäischer Ebene: das europäische Regierungssystem sei eben nicht mit dem nationalen vergleichbar und die Einflussmöglichkeiten der Europarteien noch in einer embryonalen Phase. Eine positive Ausnahme sei die Wahl des Kommissionspräsidenten Barroso gewesen, die maßgeblich von den europäischen Christdemokraten mitbestimmt wurde.

An der Schnittstelle zwischen nationalen Parteien und den Fraktionen des Europäischen Parlaments ist das Forschungsthema von Gail McElroy angesiedelt: sie fragte in ihrem Vortrag danach, an welchen Kriterien sich die Parteien bei der Fraktionswahl orientieren. ${ }^{4}$ Bislang hätte man die Parteifamilie als das entscheidende Kriterium angenommen - ein zu vager und im Falle der osteuropäischen Mitgliedsländer nicht anwendbarer Ansatz,

4 Vgl. Gail McElroy, Kenneth Benoit: Party Policy and Party Group Affiliation in the European Parliament, Trinity College Dublin, 6 July 2005, http://faculty.virginia.edu/partyswitching/papers/cv05-mcelroy-benoit.pdf (letzter Zugriff: 20.12.2005). 
wie McElroy kritisierte. Ausgehend von Expertenumfragen unter den europäischen und nationalen Abgeordneten, legte McElroy im ersten Schritt dar, dass der politische Wettbewerb auf beiden Ebenen ähnlich strukturiert ist. So bilde die Rechts-Links-Achse auch im Europäischen Parlament die Hauptdimension des politischen Wettbewerbs. Ausgehend von dieser Konvergenz hat McElroy nun die politischen Positionen der Abgeordneten beider Ebenen, in den nationalen Parlamenten und im Europäischen Parlament, einander gegenübergestellt. Dabei kam sie zum Ergebnis: Je mehr sich die Positionen einer nationalen Partei denen einer Fraktion im Europäischen Parlament auf der Rechts-Links-Achse ähneln, desto wahrscheinlicher ist der Anschluss der Partei an diese Fraktion. Interessanterweise stellte McElroy dieselbe Tendenz in abgeschwächter Form auch bei den osteuropäischen Parteien fest. Zugleich betonte sie, dass es bemerkenswerte Abweichungen von dem Schema gäbe. So seien alle drei britischen Parteien ideologisch gesehen in einer falschen Fraktion, was mit bestimmten außerpolitischen Faktoren zusammenhinge - ,the policy does not tell the entire story". Die Kosten für den Übergang der britischen Konservativen (EVP) in die ideologisch nähere, aber politisch marginale Fraktion „Unabhängigkeit und Demokratie“ (IND/DEM) seien einfach zu hoch.

\section{Instabile Parteiensysteme und, second order national elections ' in Osteuropa}

Was die Parteiensysteme der neuen Mitgliedstaaten betrifft, nahmen an der Tagung mehrere Parteienforscher, meist aus den entsprechenden Staaten, teil. In ihren Beiträgen, ebenso wie in den anschließenden Diskussionen ging es insbesondere um die Instabilität der Parteiensysteme, die Europawahlen und die Rolle der nationalen Abgeordneten im Europäischen Parlament. Evald Mikkel begann mit einem Blick auf die baltischen Parteien- systeme: hatten die ersten drei Parlamentswahlen noch Tendenzen zur Konsolidierung gezeigt, so war die vierte Wahl sowohl in Estland als auch in Litauen durch den Sieg neu gegründeter Parteien, einer grundlegenden Veränderung der bisherigen Machtverhältnisse und steigende Fragmentierung gekennzeichnet. Als ein besonderes Merkmal kleiner Gesellschaften hob Mikkel die „Kartellisierung" der estnischen Parteienlandschaft hervor: eine starke programmatische Annäherung der Parteien und die parteipolitische Durchdringung der Verwaltung führten zur Einschränkung der Wahlmöglichkeiten der Bürger. Auch wenn Jan Rovny das tschechische Parteiensystem als relativ stabil charakterisierte, sei das eine ,stability with questionmarks", geprägt durch eine (oben schon mehrmals erwähnte) Personalisierung der Politik und die Präsenz der alten postkommunistischen Führungsriege. Er prognostizierte Auswirkungen dieser Instabilitäten auch auf europäischer Ebene.

Beim Thema der Europawahl fand insbesondere die These der ,second order national elections"5 Bestätigung. So waren in Estland, Lettland und Tschechien die Oppositionsparteien siegreich, in Litauen die neugegründete Arbeitspartei. In Tschechien verursachte die Europawahl sogar den Rücktritt des Premierministers Vladimír Spidla (CSSD). Zusätzlich waren niedrige Wahlbeteiligungen und die Dominanz innenpolitischer Wahlkampfthemen zu verzeichnen.

Mit diesen Mustern korrespondiert auch die marginale Rolle der neuen Europaabgeordneten für die nationalen Mutterparteien: so berichtete Janis Ikstens, dass die Abgeordneten zwar durchaus als Europaexperten wahrgenommen werden, in der nationalen politischen Arena jedoch nur wenig zu sagen haben und von der eigenen Partei lediglich als eine „zusätzliche Finanzquelle" gesehen werden. Ähnlich bemerkte Mindaugas Jurkynas, dass

5 Vgl. Karlheinz Reif/Hermann Schmitt: Nine Second-Order National Elections - a Conceptual Framework for the Analysis of European Election Results, in: European Journal of Political Research, 8 (1980), S. 3-44. 
das Europäische Parlament von Parteien nicht als wichtige zusätzliche Aktionsarena gesehen wird; Rovny sah darin einen „Warteraum" für nationale Abgeordnete.

Relativiert werden müssen auch die Möglichkeiten der Abgeordneten der kleinen Mitgliedstaaten, nationale Anliegen auf europäischem Terrain hörbar zu machen. In diesem Zusammenhang kam man auch auf die spezifischen Interessen der russischsprachigen Minderheiten im Baltikum zu sprechen: liege hier das Potential für die Gründung einer transnationalen ethnischen Partei? Ikstens berichtete von einer Initiative der Parteien aus sechs Ländern mit Sitz in Riga; insgesamt jedoch sei ihr kultureller Hintergrund zu heterogen, um eine längerfristige Zusammenarbeit zu begründen.

Von einem sich von den osteuropäischen deutlich unterscheidenden Parteiensystem berichtete Peter Zervakis: Die Europawahlkampagne in Zypern sei von dem missglückten Referendum zur Vereinigung der Insel überschattet worden und hätte in politischer Hinsicht keine Überraschungen gebracht. Im Europäischen Parlament sei jedoch ein Engagement der zypriotischen Abgeordneten gegen die Zypernpolitik der Türkei zu erwarten.

Eine neue Entwicklungsstufe der Europarteien?

Der Themenblock zur zweiten Dimension des europäischen Parteienwettbewerbs - zu den Europarteien - wurde von Robert Ladrech und Luciano Bardi eingeleitet, die in einer top-down-Perspektive nach dem Einfluss von Europa auf die Organisationsstruktur der nationalen Parteien fragten. Da diese die Basis der europäischen Parteien bilden, bedeutet eine Aussage über ihre Europäisierungsstufe zugleich ein qualitatives Urteil über den Entwicklungsfortschritt der europäischen Parteien selbst. In diesem Sinne zeugt das eher ernüchternde Ergebnis von Robert Ladrech von keinen guten Aussichten für die weitere Parteipolitisierung des europäischen Regie- rungssystems. Laut seiner Feldstudie zu Parteien aus sechs Mitgliedstaaten (Deutschland, Großbritannien, Frankreich, Schweden, Spanien, Österreich) gab es nur marginale Veränderungen der inneren Organisationsstruktur der Parteien, wie beispielsweise die Einführung des Postens eines „Europasekretärs“. Der innerparteiliche Einfluss der mit Europapolitik befassten Politiker sei während der Mitgliedschaft dieser Parteien in europäischen Parteistrukturen nicht gewachsen, was zugleich eine große Autonomie und geringe Verantwortlichkeit der Europapolitiker gegenüber den nationalen Parteieliten bedeute. Als Gründe für diese Ergebnisse nannte Ladrech die Wahrnehmung der auf supranationaler Ebene tätigen Politiker als „Auslandsarbeiter"; die Europapolitik werde in Netzwerken aus Europaexperten und Beamten „außerhalb“ der eigentlichen Partei gemacht. Auch seien die Parteieliten an einer aktiven Mitarbeit bei den Europarteien nicht interessiert, um die eigene Autonomie möglichst ungeschmälert zu erhalten. Einen vergleichsweise geringen Einfluss auf die italienischen Parteien konstatierte auch Luciano Bardi: zwar hätten die Europapolitiker beschränkt an Einfluss gewonnen, nichtsdestotrotz behielte die nationale Parteiführung das letzte Wort.

Nach diesem sehr ernüchternden Urteil zum Europäisierungsstand der nationalen Parteien wandte sich David Hanley der Frage zu, welche Konsequenzen die Erweiterungspolitik der Union für die Europarteien hat(te). Ähnlich wie zuvor Lang kam Hanley zu dem Schluss, dass die Europarteien die Osterweiterung insgesamt sehr erfolgreich gemeistert haben, was den Lernprozessen in früheren Erweiterungsrunden zu danken sei. Insbesondere die Nutzbarmachung der Erfahrungen mit Parteien postautoritärer Systeme bei der Süderweiterung lieferte den Europarteien das nötige Know-how für die neueste Erweiterungsrunde. Nicht zu unterschätzen sei dabei die Rolle der politischen Stiftungen, die wichtige Arbeit bei der Konsolidierung der Parteien und somit der Parteiensysteme leisteten. Dennoch sei die Osterweiterung von den Eu- 
roparteien am besten und am längsten vorbereitet worden - die Kontakte zu osteuropäischen Parteien wurden gleich nach dem Verschwinden des Eisernen Vorhangs aufgebaut. Ideologisch am einfachsten schätzte Hanley die Position der Europäischen Sozialisten ein, für die der Erweiterungsprozess durch den „Recyclingprozess“ der Exkommunisten gradlinig verlaufen sei. Dagegen wären die europäischen Grünen die Verlierer der Osterweiterung gewesen. Alles in allem hätte die letzte Erweiterung insbesondere die Reihen der Souveränisten (wie die Fraktion „Union für das Europa der Nationen“/UEN) und der kleineren Europarteien gestärkt - ein typischer Fall von bottom-up-Europäisierung. Umgekehrt kann die erfolgreiche Integrationsstrategie der großen Europarteien wie SPE, EVP und ELDR als ein Beispiel für eine erfolgreiche top-down-Europäisierung angesehen werden.

Im Anschluss referierte Rudolf Hrbek über Entwicklungen im Zusammenhang mit dem europäischen Parteienstatut. Seit dem „Parteienartikel“ im Vertrag von Maastricht, der erstmals die Rolle und Relevanz europäischer Parteien unterstreicht, gab es eine kontinuierliche Weiterentwicklung des legalen Status der Europarteien. Als Zwischenstation nannte Hrbek den Nizza-Vertrag, der Regelungen für die europäischen Parteien und ihre Finanzierung als eine Aufgabe des Europäischen Rats festschrieb, bevor es im November 2003 zur Verabschiedung des europäischen Parteienstatuts kam. Dieses wurde unter großem (finanziellen) Druck auf den Weg gebracht, denn vor Jahren hatte der Europäische Rechnungshof die unrechtmäßige Mitfinanzierung der Europarteien durch die Fraktionen des Europäischen Parlaments gerügt. Hrbek vertrat die Auffassung, dass die europäischen Parteien bereits den Maastrichter Parteienartikel vor allem mit der Perspektive auf die künftige Finanzierung aus dem Haushalt der Union angestoßen hätten. Dieses Ziel sei nun mit dem Parteienstatut umgesetzt worden: neben einer detaillierten Definition einer „Partei auf europäischer Ebene" (so die Formulierung im Parteienstatut) legt das Statut die Einzelheiten der Finanzierung der Europarteien fest. Hrbek vermutete, dass die Festlegung der Finanzierungsregeln durch das Statut Ansporn für die Gründung neuer transnationaler Parteien gewesen sei. ${ }^{6}$ Während Hrbek das Parteienstatut ganz generell als eine Wegmarke bei der Entwicklung eines europäischen Parteiensystems betrachtete, kritisierten die Parteienvertreter Kremer und Helle in der anschließenden Diskussion das Dokument als nur bedingt gute Grundlage für die praktische Arbeit der Europarteien. Die Vorgabe, die Registrierung einer Europartei im Staat ihres Vorsitzes vornehmen zu müssen, schränke aufgrund der Besonderheiten des belgischen Rechts die Parteien ein: dies sehe nämlich keinen eigenen rechtlichen Status für Parteien vor.

Eine (Partei-)Politisierung des europäischen Regierens?

Der Titel des letzten Themenblocks „Parteien als Faktor in den EU-Institutionen und in interinstitutionellen Beziehungen" zielte auf die parteipolitische Durchdringung des europäischen Regierungssystems und damit auf eine mögliche Parallele mit nationalen Regierungssystemen. Diese Parallele wurde von Christopher Lord jedoch verneint: das klassische Modell der parteipolitischen Verantwortlichkeit der Regierung sei nicht auf das europäische Regierungssystem zu übertragen. Dennoch ist es durchaus bemerkenswert, dass die Europarteien es geschafft haben, ihren Einfluss auch über das Europäische Parlament hinaus auszudehnen. Die Einflussmöglichkeiten seien jedoch je nach Institution unterschiedlich. So hätten die meisten von den nationalen Regierungen bestimmten Kommissare einen Parteihintergrund, auch wenn die Einflussmöglichkeiten der Europarteien auf die Kommission als relativ gering einzuschätzen sind. Die Tatsache, dass einzelne Kommissare keine Bestätigung durch das Europäi-

6 Z.B. wurden im Jahr 2004 die Europäische Demokratische Partei und die Europäische Linkspartei gegründet. 
sche Parlament erhielten, werfe die Frage nach der Autonomie des Parlaments auf: inwiefern stecken parteipolitische Überlegungen und Absprachen hinter diesen Abstimmungsergebnissen? Im Fall des Rats ist der reale Einfluss der europäischen Parteien relativ schwer einzuschätzen, wird aber wohl vor allem im Vorfeld der Treffen des Europäischen Rats und der Ratsformationen sichtbar: hier kommt es zu regelmäßigen formalisierten Meetings von nationalen Regierungsmitgliedern mit den Vorsitzenden der entsprechenden nationalen Parteien und den Führern der europäischen Partei (Fraktion und Parteiorganisation). Insgesamt betonte Lord, dass das europäische Institutionensystem die Rolle der Parteien neutralisiert, da die Entscheidungsfindung meistens nur auf einer breiten Basis und über mehrere Parteienfamilien hinweg möglich ist.

Zuletzt ging Andreas Helle auf die spezielle Art der Ausarbeitung gemeinsamer Positionen innerhalb der europäischen „Mehrebenenparteien" ein. Zu diesen mehr oder weniger formalisierten Formen der Entscheidungsfindung zählten neben den traditionellen innerparteilichen Organen (Präsidium, Kongress etc.) auch zeitlich befristete Einrichtungen. Die Treffen der ad hoc-Arbeitsgruppen, bestehend aus Vertretern interessierter Mitgliedsparteien und dem Präsidium, finden drei- bis viermal jährlich statt, um gemeinsame Vorschläge vorzubereiten. Zur Erarbeitung der working papers dienen sogenannte high level groups. Als Beispiel für breiter angelegte network groups innerhalb der SPE nannte Helle Arbeitsgruppen zu Fragen einer europäischen Verfassung und zur Mittelmeerkooperation. Insgesamt seien nationale Oppositionsparteien bei diesen gemeinsamen Aktionen wesentlich aktiver, da sie die europäische Ebene als zusätzliche Aktionsebene wahrnehmen. Alle diese Arbeitsformen seien typische Beispiele für die Organisation gemeinsamer Aktivitäten in europäischen Mehrebenenparteien.

Die wesentlichen Ergebnisse der Tagung können in folgenden drei Punkten zusammengefasst werden:

- Die Entwicklung der europäischen Parteien ist im Kontext der Vertiefung der Union zu sehen; die Konstitutionalisierung hat auch die europäischen Parteien nicht unberührt gelassen - erkennbar insbesondere an der Weiterentwicklung ihrer institutionellen Grundlagen.

- Auch wenn die Osterweiterung eine beachtliche Erweiterung der ideologischen und Mitgliederbasis der europäischen Parteien mit sich gebracht hat, ist sie auf der Grundlage der Lernprozesse bei früheren Erweiterungen erfolgreich verarbeitet worden.

- Das europäische Parteiensystem ist insbesondere und ausschließlich vor dem Hintergrund der vielfältigen und komplexen Wechselbeziehungen zwischen nationalen Parteien, den EP-Fraktionen und den Führungen der transnationalen Parteiorganisationen in Brüssel zu verstehen und zu erforschen.

Es ging bei der Tagung nicht um die Frage, ob es ,Europäische“ Parteien gibt. Vielmehr galt es zu erörtern, auf welcher Entwicklungsstufe sie angelangt sind und welche Kontextfaktoren ihre Arbeit beeinflussen. Um den Ausdruck eines Konferenzteilnehmers zu verwenden: die europäischen Parteien sind als eine Art ,Multi-Level-Parteien ' anzusehen, deren Funktionsweise stark durch die spezielle Art des europäischen Regierungssystems bestimmt wird. 